\title{
ON ASYMPTOTIC APPROXIMATIONS OF SOLUTIONS OF AN EQUATION WITH A SMALL PARAMETER
}

\author{
A. M. IL'IN AND E. F. LELIKOVA
}

Dedicated to our teacher Vasiliı Mikhaĭlovich Babich

\begin{abstract}
A second order elliptic equation with a small parameter at one of the highest order derivatives is considered in a three-dimensional domain. The limiting equation is a collection of two-dimensional elliptic equations in two-dimensional domains depending on one parameter. By the method of matching of asymptotic expansions, a uniform asymptotic approximation of the solution of a boundary-value problem is constructed and justified up to an arbitrary power of a small parameter.
\end{abstract}

\section{$\S 1$. INTRODUCTION}

Problems for elliptic equations with small parameters at highest order derivatives are the subject of intense studies. We mention only the earliest papers [1]-4] on this subject and the extensive survey [5. To treat more complicated problems of this type, we need to exploit the method of matching of asymptotic expansions, also called the matching method. In [6] -8 and [10] this method was developed and applied to various problems, including elliptic equations.

In the majority of papers on elliptic equations with small parameters at highest derivatives, the reduction of order is applied, in which the limiting equation has an order smaller than the perturbed equation. In particular, this was the reason for the singularity of the problem. At the same time, it turns out that even if the order reduction is not used, the problem often continues to be singular, and more involved methods are necessary for its solution.

Some cases in which the limiting equation is an ordinary differential equation were considered in the recent papers [1]-13.

In the present paper, we also assume that the limiting equation is a second order elliptic equation but with a smaller number of independent variables. We consider the perturbed equation in a three-dimensional domain. The limiting equation is a collection of two-dimensional equations in domains depending on one parameter. The asymptotic behavior of a solution of a boundary-value problem depends substantially on the structure of the domain at singular points related to the degeneration type of the operator. In this regard, the situation is similar to the case where degeneration causes order reduction. In general, our method is close to that used in the above-mentioned cases. However, the auxiliary problems that arise in passing require other methods. Sometimes, they are more involved. Even the formal asymptotic expansion is not constructed in all cases of

2010 Mathematics Subject Classification. Primary 35J47.

Key words and phrases. Asymptotic, boundary value problem, small parameter, matching of asymptotic expansions.

Supported by RFBR (grants nos. 08-01-00260, 09-01-00530), by a grant NSh-6249.2010.1, and by a grant FTsP 02.740.110612. 
general position. Here, we study in detail the case where, in a neighborhood of a singular point, the domain of the perturbed problem lies on one side of the tangent plane and the limiting problems are considered in two-dimensional contracting domains.

\section{§2. Statement of the PRoblem and external EXPANSION OF THE SOLUTION}

We consider the first boundary-value problem for the equation

$$
\begin{aligned}
\mathbb{M}_{\varepsilon} u & =\varepsilon u_{z z}+\mathrm{L} u=f(x, y, z), \\
u(x, y, z) & =0, \quad(x, y, z) \in \partial D,
\end{aligned}
$$

in a bounded domain $D \subset \mathbb{R}^{3}$. Here, L is an elliptic operator,

$$
\begin{aligned}
\mathrm{L} u= & a_{11}(x, y, z) \frac{\partial^{2} u}{\partial x^{2}}+2 a_{12}(x, y, z) \frac{\partial^{2} u}{\partial x \partial y}+a_{22}(x, y, z) \frac{\partial^{2} u}{\partial y^{2}} \\
& +b_{1}(x, y, z) \frac{\partial u}{\partial x}+b_{2}(x, y, z) \frac{\partial u}{\partial y}+c(x, y, z) u .
\end{aligned}
$$

We assume that $\varepsilon>0, c(x, y, z) \leq 0$, and all coefficients of equation (11) and the righthand side are infinitely differentiable.

It is well known that, under these assumptions, there exists a bounded solution of problem (11), (2), which we denote by $u_{\varepsilon}(x, y, z)$, satisfying the estimate $\left|u_{\varepsilon}(x, y, z)\right| \leq$ $M \max _{(x, y, z) \in D}|f(x, y, z)|$, where the constant $M$ does not depend on $\varepsilon$.

We assume that $\partial D$ (the boundary of $D$ ) is infinitely differentiable. For simplicity, we assume that the domain $D$ is convex and the planes $z=$ const are tangent to $\partial D$ only at two points $\mathbb{P}_{0}$ and $\mathbb{P}_{1}$, where $\mathbb{P}_{0}$ is the origin and $\mathbb{P}_{1}=\left(x_{1}, y_{1}, z_{1}\right), z_{1}>0$.

To describe the results obtained, we introduce some notation. We denote by $\Omega_{z}$ the intersection of $D$ with the plane $z=$ const and by $\partial \Omega_{z}$ the boundary of this intersection.

We shall construct the standard external expansion of the solution $u_{\varepsilon}(x, y, z)$ as $\varepsilon \rightarrow 0$ in the form

$$
u_{\varepsilon}(x, y, z)=U(x, y, z, \varepsilon)=\sum_{k=0}^{\infty} \varepsilon^{k} u_{k}(x, y, z) .
$$

Substituting (3) into (11) and equating coefficients at the same powers of $\varepsilon$, we obtain the recurrence relations

$$
\begin{aligned}
& \mathrm{L} u_{0}=f(x, y, z), \quad(x, y) \in \Omega_{z}, \\
& \mathrm{~L} u_{k}=-\frac{\partial^{2} u_{k-1}}{\partial z^{2}}, \quad k \geq 1, \quad(x, y) \in \Omega_{z} .
\end{aligned}
$$

By the boundary condition (2) of our problem, we obtain the following boundary conditions for the functions $u_{k}(x, y, z)$ at a fixed point $z$ on the boundary of the plane domain $\Omega_{z}$ :

$$
u_{k}(x, y, z)=0, \quad k \geq 0, \quad(x, y) \in \partial \Omega_{z} .
$$

Thus, for every fixed $z$, the functions $u_{k}(x, y, z)$ are solutions of the two-dimensional recurrence problems (4) and (5) in the domain $\Omega_{z}$ lying in planes parallel to the plane $(x 0 y)$, and the size of the domain $\Omega_{z}$ tends to zero as $z \rightarrow+0$ and $z \rightarrow z_{1}-0$. The domains $\Omega_{z}$ "shrink" to the points $\mathbb{P}_{0}$ and $\mathbb{P}_{1}$.

The variable $z$ in the function $u_{k}(x, y, z)$ is regarded as a parameter, and it turns out that, in general, these functions have singularities at the points $\mathbb{P}_{i}$, the order of a singularity increases with $k$, and, thus, the external expansion (3) becomes useless in neighborhoods of these points. In neighborhoods of $\mathbb{P}_{i}$, we construct another asymptotic expansion. For this, we use the matching method [10. In more detail, we study the 
behavior of the solution in a neighborhood of the origin. The case of a neighborhood of the point $\mathbb{P}_{1}$ is studied similarly. The situation depends crucially on the structure of the boundary in a neighborhood of the point at which the boundary touches the plane. It turns out that if the curvature of the surface at this point is nonzero, then the coefficients of the external expansion have no singularities. Not dwelling on this here (see the remark at the end of the paper), we consider in detail the case where the problem is bisingular, i.e., the coefficients $u_{k}(x, y, z)$ have growing singularities as $z \rightarrow+0$. We assume that the equation of the boundary $\partial \Omega_{z}$ has the form $z=x^{4}+y^{4}$ for $z \leq \delta$, where $\delta>0$ is a fixed number.

Let $D_{\delta}=\{(x, y, z) \in D, 0<z<\delta\}$. In this fixed neighborhood of the origin, we construct an asymptotic expansion of the solution $u_{\varepsilon}$ as $\varepsilon \rightarrow+0$.

To study the asymptotics of the coefficients $u_{k}(x, y, z)$ as $z \rightarrow+0$, i.e., in a neighborhood of the origin, we expand the right-hand side $f(x, y, z)$ and the coefficients of the operator L in Taylor series. Taking into account the structure of the domain in a neighborhood of the origin, we group the terms of the Taylor series into generalized homogeneous polynomials, setting the degree of the variable $z$ equal to four times the degrees of the variables $x$ and $y$. For a smooth function $v(x, y, z)$, such a representation has the form

$$
\left\{\begin{array}{l}
v(x, y, z)=\sum_{k=0}^{\infty} P_{k}(x, y, z), \\
P_{k}(x, y, z)=\sum_{j=0}^{\left[\frac{k}{4}\right]} q_{k-4 j}(x, y) z^{j},
\end{array}\right.
$$

and $q_{m}(x, y)$ are homogeneous polynomials in $x$ and $y$ of degree $m$.

We change the variables $x$ and $y$, putting

$$
\xi=x z^{-\frac{1}{4}}, \quad \eta=y z^{-\frac{1}{4}} .
$$

In the new variables the polynomials $P_{k}(x, y, z)$ have the form

$$
P_{k}(x, y, z)=z^{\frac{k}{4}} \sum_{j=0}^{\left[\frac{k}{4}\right]} q_{k-4 j}(\xi, \eta) \equiv z^{\frac{k}{4}} R_{k}(\xi, \eta) .
$$

In the operator $\mathrm{L}$, we replace the functions $a_{i j}(x, y, z)$ by series of the form (6), (8), obtaining

$$
\left\{\begin{array}{l}
a_{i j}(x, y, z)=a_{i j}(0,0,0)+\sum_{k=1}^{\infty} z^{\frac{k}{4}} A_{k, i j}(\xi, \eta), \\
b_{j}(x, y, z)=\sum_{k=0}^{\infty} z^{\frac{k}{4}} B_{k, j}(\xi, \eta), \\
c(x, y, z)=\sum_{k=0}^{\infty} z^{\frac{k}{4}} C_{k}(\xi, \eta) .
\end{array}\right.
$$

Now, we pass from the variables $x$ and $y$ to the variables $\xi$ and $\eta$. Denote

$$
\begin{aligned}
\mathrm{L}_{0}= & a_{11}(0,0,0) \frac{\partial^{2}}{\partial \xi^{2}}+2 a_{12}(0,0,0) \frac{\partial^{2}}{\partial \xi \partial \eta}+a_{22}(0,0,0) \frac{\partial^{2}}{\partial \eta^{2}}, \\
\mathrm{~L}_{m}= & A_{m, 11}(\xi, \eta) \frac{\partial^{2}}{\partial \xi^{2}}+2 A_{m, 12}(\xi, \eta) \frac{\partial^{2}}{\partial \xi \partial \eta}+A_{m, 22}(\xi, \eta) \frac{\partial^{2}}{\partial \eta^{2}} \\
& +B_{m-1,1}(\xi, \eta) \frac{\partial}{\partial \xi}+B_{m-1,2}(\xi, \eta) \frac{\partial}{\partial \eta}+C_{m-2}(\xi, \eta), \quad m>0,
\end{aligned}
$$

where $C_{-1} \equiv 0$.

We represent the operator $\mathrm{L}$ in the form

$$
\mathrm{L}=z^{-\frac{1}{2}} \mathrm{~L}_{0}+\sum_{j=1}^{\infty} z^{\frac{j-2}{4}} \mathrm{~L}_{j}
$$


Theorem 1. There exist unique solutions $u_{k}(x, y, z)$ of problems (44), (5) that are infinitely differentiable in $x$ and $y$. As $z \rightarrow+0$, we have the following asymptotic expansions for $u_{k}(x, y, z)$ :

$$
u_{k}(x, y, z)=\sum_{j=2-6 k}^{\infty} z^{\frac{j}{4}} u_{k j}\left(x z^{-\frac{1}{4}}, y z^{-\frac{1}{4}}\right),
$$

where the functions $u_{k j}(\xi, \eta)\left(\xi=x z^{-\frac{1}{4}}, \eta=y z^{-\frac{1}{4}}\right)$ are infinitely differentiable in the domain $\xi^{4}+\eta^{4} \leq 1$. Relation (12) admits termwise differentiation with respect to the parameter $z$.

Proof. Consider the function $u_{0}(x, y, z)$. Relations (4) and (5) show that this function solves the problem

$$
\begin{aligned}
& \mathrm{L} u_{0}=f(x, y, z), \quad(x, y, z) \in \Omega_{z}, \quad 0<z<\delta \\
& u_{0}(x, y, z)=0, \quad(x, y, z) \in \partial \Omega_{z}, \quad 0<z<\delta .
\end{aligned}
$$

We construct an asymptotic representation of the function $u_{0}(x, y, z)$ in the form of the series (12), i.e.,

$$
u_{0}(x, y, z)=\sum_{j=2}^{\infty} z^{\frac{j}{4}} u_{0 j}(\xi, \eta) .
$$

Also, we represent the Taylor series of $f(x, y, z)$ in a form similar to (9),

$$
f(x, y, z)=\sum_{k=0}^{\infty} \widetilde{P}_{k}(x, y, z)=\sum_{k=0}^{\infty} z^{\frac{k}{4}} \widetilde{q}_{k}(\xi, \eta) .
$$

Using relation (16) and formula (11) for the operator L, we substitute the asymptotic expansion under consideration in equation (13). Equating the terms with the same powers of $z$, we obtain recurrence relations for the required functions $u_{0 j}(\xi, \eta)$,

$$
\left\{\begin{array}{l}
\mathrm{L}_{0} u_{02}=\widetilde{q}_{0}(\xi, \eta), \\
\mathrm{L}_{0} u_{03}=-\mathrm{L}_{1} u_{02}+\widetilde{q}_{1}(\xi, \eta), \\
\mathrm{L}_{0} u_{0 m}=-\sum_{j=1}^{m-2} \mathrm{~L}_{j} u_{0, m-j}+\widetilde{q}_{m-2}(\xi, \eta), m>3 .
\end{array}\right.
$$

The boundary condition (14) for the series (15) takes the form

$$
u_{0 j}(\xi, \eta)=0
$$

for $\xi^{4}+\eta^{4}=1$.

Thus, we define the functions $u_{0 j}(\xi, \eta)$ as the solutions of problems (17) and (18). It is well known (see, e.g., 14, 15) that the solutions of these problems exist and are infinitely differentiable in $\xi$ and $\eta$.

To justify the asymptotic formula (15), we proceed in the standard way. We consider a partial sum

$$
S_{N}(x, y, z)=\sum_{j=2}^{N} z^{\frac{j}{4}} u_{0, j}(\xi, \eta),
$$

where $N$ is a sufficiently large number. By construction, we have

$$
\begin{aligned}
\mathrm{L}\left(u_{0}(x, y, z)-S_{N}(x, y, z)\right) & =O\left(z^{\frac{N-1}{4}}\right), \quad(x, y, z) \in \Omega_{z}, \\
u_{0}(x, y, z)-S_{N}(x, y, z) & =0, \quad(x, y, z) \in \partial \Omega_{z} .
\end{aligned}
$$

Consequently, since $c(x, y, z) \leq 0$, we obtain

$$
\left|\left(u_{0}(x, y, z)-S_{N}(x, y, z)\right)\right| \leq M z^{\frac{N-1}{4}}, \quad(x, y, z) \in \Omega_{z} .
$$


The fact that formula (12) admits termwise differentiation with respect to the parameter $z$ follows from the well-known internal estimates for the derivatives of solutions of elliptic equations and from estimates for these derivatives in the vicinity of the smooth boundary; see [14. Although the boundary of the domain itself depends on the parameter $z$, it turns into the domain $\xi^{4}+\eta^{4}<1$ after the change of variables (17). The function $f(x, y, z)$ turns into the function $\widetilde{f}\left(\xi z^{\frac{1}{4}}, \eta z^{\frac{1}{4}}, z\right)$. The function $u_{0}(x, y, z)$ changes in a similar way. By construction, the difference between the derivative of $u_{0}(x, y, z)$ and the derivative of the partial sum $S^{N}(x, y, z)$ satisfies an equation the right-hand side of which is small for small $z$. Since we have proved that the difference itself is small for small $z$, the abovementioned estimates for derivatives imply that the absolute value of the difference of the corresponding derivatives also does not exceed a high power of $z$ if the partial sum is sufficiently long, regardless of what variables we use, $(x, y, z)$ or $(\xi, \eta, z)$, to calculate the derivative.

Applying the same argument to the higher derivatives, we see that the series admits termwise differentiation of an arbitrary order. This proves the claim of Theorem 1 for the function $u_{0}(x, y, z)$.

Now, we consider the function $u_{1}(x, y, z)$. It satisfies the equation

$$
\mathrm{L} u_{1}=-\frac{\partial^{2} u_{0}}{\partial z^{2}}=F_{1}(x, y, z), \quad(x, y, z) \in \Omega_{z},
$$

and vanishes on the boundary $\omega_{z}$ of $\Omega_{z}$.

As has been proved above, the function $F_{1}(x, y, z)$ has the asymptotic expansion

$$
F_{1}(x, y, z)=\sum_{j=-6}^{\infty} z^{\frac{j}{4}} f_{j}(\xi, \eta) .
$$

It is easy to obtain explicit expressions for $f_{j}(\xi, \eta)$ in terms of the function $u_{0, j+8}(\xi, \eta)$ and its first and second order derivatives.

As in the study of the function $u_{0}(x, y, z)$, first we construct a formal asymptotic expansion (12) for the function $u_{1}(x, y, z)$. The functions $u_{1 j}(\xi, \eta)$ are found as solutions of problems of the form (17), (18),

$$
\left\{\begin{array}{l}
\mathrm{L}_{0} u_{1,-4}=f_{-6}(\xi, \eta) \\
\mathrm{L}_{0} u_{1,-3}=-\mathrm{L}_{1} u_{1,-4}+f_{-5}(\xi, \eta), \\
\mathrm{L}_{0} u_{1, m}=-\sum_{j=1}^{m+4} \mathrm{~L}_{j} u_{1, m-j}+f_{m-2}(\xi, \eta), \quad m>-3,
\end{array}\right.
$$

$u_{0 j}(\xi, \eta)=0$ for $\xi^{4}+\eta^{4}=1$.

The further details are the same as those for the function $u_{0}(x, y, z)$; thus, the claim is proved for the function $u_{1}(x, y, z)$.

The asymptotic expansions (12) as $z \rightarrow+0$ for the functions $u_{k}(x, y, z), k \geq 2$, are constructed and justified similarly.

Obviously, the functions $u_{k}(x, y, z)$ have singularities as $z \rightarrow+0$, and the order of these singularities grows with $k$. Therefore, the asymptotic expansion (3) is certainly invalid for small $z$. In a neighborhood of zero, we must seek a different form of the asymptotic expansion for the solution of problem (11), (2).

\section{§3. INTERNAL EXPANSION}

In a neighborhood of the origin, we pass to the new, internal variables

$$
\sigma=x \varepsilon^{-\frac{1}{6}}, \quad \tau=y \varepsilon^{-\frac{1}{6}}, \quad \zeta=z \varepsilon^{-\frac{2}{3}}
$$


and construct an internal asymptotic expansion of the solution $u_{\varepsilon}(x, y, z)$ in the form

$$
u_{\varepsilon}(x, y, z)=V(x, y, z, \varepsilon)=\sum_{i=2}^{\infty} \varepsilon^{\frac{i}{6}} v_{i}(\sigma, \tau, \zeta) .
$$

As in the case of the external expansion, we group the terms of the Taylor series for the coefficients of the equation and for the right-hand side into generalized homogeneous polynomials, setting the degree of the variable $z$ equal to four times the degrees of the variables $x$ and $y$. For a smooth function $v(x, y, z)$, such a representation is given by formulas (6) with the subsequent change of variables (19). Continuing formula (8), we obtain

$$
P_{k}(x, y, z)=P_{k}\left(\sigma \varepsilon^{\frac{1}{6}}, \tau \varepsilon^{\frac{1}{6}}, \zeta \varepsilon^{\frac{2}{3}}\right)=\varepsilon^{\frac{k}{6}} Z_{k}(\sigma, \tau, \zeta) .
$$

After these transformations, we represent the operator $\mathbb{M}_{\varepsilon}$ in the form

$$
\mathbb{M}_{\varepsilon}=\varepsilon^{-\frac{1}{3}}\left(\mathrm{M}_{0}+\sum_{j=1}^{\infty} \varepsilon^{\frac{j}{6}} \mathrm{M}_{j}\right)
$$

where

$$
\begin{aligned}
\mathrm{M}_{0}= & \frac{\partial^{2}}{\partial \zeta^{2}}+a_{11}(0,0,0) \frac{\partial^{2}}{\partial \sigma^{2}}+2 a_{12}(0,0,0) \frac{\partial^{2}}{\partial \sigma \partial \tau}+a_{22}(0,0,0) \frac{\partial^{2}}{\partial \tau^{2}}, \\
\mathrm{M}_{m}= & \alpha_{m, 11}(\sigma, \tau, \zeta) \frac{\partial^{2}}{\partial \sigma^{2}}+2 \alpha_{m, 12}(\sigma, \tau, \zeta) \frac{\partial^{2}}{\partial \sigma \partial \tau}+\alpha_{m, 22}(\sigma, \tau, \zeta) \frac{\partial^{2}}{\partial \tau^{2}} \\
& +\beta_{m-1,1}(\sigma, \tau, \zeta) \frac{\partial}{\partial \sigma}+\beta_{m-1,2}(\sigma, \tau, \zeta) \frac{\partial}{\partial \tau}+\gamma_{m-2}(\sigma, \tau, \zeta), \quad m>0 .
\end{aligned}
$$

We also represent the right-hand side of equation (10) in the form

$$
f(x, y, z)=\sum_{k=0}^{\infty} \widetilde{P}_{k}(x, y, z)=\sum_{k=0}^{\infty} \varepsilon^{\frac{k}{6}} Q_{k}(\sigma, \tau, \zeta),
$$

where $Q_{k}(\sigma, \tau, \zeta)=O\left(\zeta^{\frac{k}{4}}\right)$.

Using the above representation of $f(x, y, z)$ and the operator $\mathbb{M}_{\varepsilon}$, we can transform (11) into the equation

$$
\varepsilon^{-\frac{1}{3}}\left(\mathrm{M}_{0}+\sum_{j=1}^{\infty} \varepsilon^{\frac{j}{6}} \mathrm{M}_{j}\right) u_{\varepsilon}=\sum_{k=0}^{\infty} \varepsilon^{\frac{k}{6}} Q_{k}(\sigma, \tau, \zeta) .
$$

Substituting the series (20) into this equation and comparing the coefficients of the same powers of $\varepsilon$, we obtain the following system of recurrence relations for the functions $v_{k}(\sigma, \tau, \zeta):$

$$
\left\{\begin{array}{l}
\mathrm{M}_{0} v_{2}=Q_{0}(\sigma, \tau, \zeta), \\
\mathrm{M}_{0} v_{3}=-\mathrm{M}_{1} v_{2}+Q_{1}(\sigma, \tau, \zeta), \\
\mathrm{M}_{0} v_{m}=-\sum_{j=1}^{m-2} \mathrm{M}_{j} v_{m-j}+Q_{m-2}(\sigma, \tau, \zeta), \quad m>3 .
\end{array}\right.
$$

In the variables $\sigma, \tau$, and $\zeta$, the boundary condition (2) takes the form

$$
v_{k}(\sigma, \tau, \zeta)=0
$$

where $\zeta=\sigma^{4}+\tau^{4}$.

Before constructing the functions $v_{k}(\sigma, \tau, \zeta)$, we find an appropriate form for their asymptotic expansions as $\zeta \rightarrow \infty$. 
In what follows, it is convenient to have a special notation for a partial sum of an asymptotic expansion. For example, for series (3), we put

$$
\mathbb{A}_{N, x, y, z} U=\sum_{j=0}^{N} \varepsilon^{k} u_{k}(x, y, z),
$$

where $N$ is a sufficiently large positive integer. Thus, the result of the application of the operator $\mathbb{A}_{N, x, y, z}$ to a series $U$ is the sum of terms of $U$ for which the exponent $k$ does not exceed $N$. It is essential that the coefficients of the series depend on $x, y$, and $z$.

Now we replace the functions $u_{k}(x, y, z)$ by their asymptotic expansions (12) as $z \rightarrow$ +0 , obtaining

$$
\mathbb{A}_{N, x, y, z} U=\sum_{k=0}^{N} \varepsilon^{k}\left[\sum_{j=2-6 k}^{\infty} z^{\frac{j}{4}} u_{k j}\left(x z^{-\frac{1}{4}}, y z^{-\frac{1}{4}}\right)\right] .
$$

Writing the right-hand side of this relation in terms of the variables $\sigma, \tau$, and $\zeta$ and taking into account formulas (19) and the relations $x z^{-\frac{1}{4}}=\sigma \zeta^{-\frac{1}{4}}$ and $y z^{-\frac{1}{4}}=\tau \zeta^{-\frac{1}{4}}$, we get

$$
\mathbb{A}_{N, x, y, z} U=\sum_{k=0}^{N} \varepsilon^{k}\left[\sum_{j=2-6 k}^{\infty} \zeta^{\frac{j}{4}} \varepsilon^{\frac{j}{6}} u_{k j}\left(\sigma \zeta^{-\frac{1}{4}}, \tau \zeta^{-\frac{1}{4}}\right)\right] .
$$

We apply the operator $\mathbb{A}_{m, \sigma, \tau, \zeta}$ defined above once again, with the only difference that now the coefficients of the series depend on $\sigma, \tau$, and $\zeta$. This yields

$$
\mathbb{A}_{m, \sigma, \tau, \zeta} \mathbb{A}_{N, x, y, z} U=\sum_{i=2}^{6 m} \varepsilon^{\frac{i}{6}}\left[\sum_{k=0}^{N} \zeta^{\frac{i-6 k}{4}} u_{k, i-6 k}\left(\sigma \zeta^{-\frac{1}{4}}, \tau \zeta^{-\frac{1}{4}}\right)\right] .
$$

It is clear that the internal sums on the right-hand side of this formula must coincide with the partial sums of the asymptotic series of the required functions $v_{i}(\sigma, t, \zeta)$ as $\zeta \rightarrow \infty$.

Let

$$
v_{i, N}(\sigma, \tau, \zeta)=\sum_{k=0}^{N} \zeta^{\frac{i-6 k}{4}} u_{k, i-6 k}\left(\sigma \zeta^{-\frac{1}{4}}, \tau \zeta^{-\frac{1}{4}}\right)
$$

and let $\widetilde{v}_{i}(\sigma, t, \zeta)$ be a temporary notation for the formal series

$$
\widetilde{v}_{i}(\sigma, \tau, \zeta)=\sum_{k=0}^{\infty} \zeta^{\frac{i-6 k}{4}} u_{k, i-6 k}\left(\sigma \zeta^{-\frac{1}{4}}, \tau \zeta^{-\frac{1}{4}}\right) \quad \text { as } \quad \zeta \rightarrow \infty .
$$

Now we denote by $\widetilde{V}(x, y, z, \varepsilon)$ the formal series

$$
\widetilde{V}(x, y, z, \varepsilon)=\sum_{i=2}^{\infty} \varepsilon^{\frac{i}{6}} \widetilde{v}_{i}(\sigma, \tau, \zeta) .
$$

Then, by construction, the following important relation is valid for all sufficiently large positive integers $N$ :

$$
\mathbb{A}_{m, \sigma, \tau, \zeta} \mathbb{A}_{N, x, y, z} U=\mathbb{A}_{N, x, y, z} \mathbb{A}_{m, \sigma, \tau, \zeta} \tilde{V}
$$

To complete the construction and justification of the asymptotic expansion of the solution $u_{\varepsilon}(x, y, z)$, it remains to prove that there exist functions $v_{i}(\sigma, \tau, \zeta)$ that satisfy system (21) and have the asymptotic expansions (23) as $\zeta \rightarrow \infty$.

Theorem 2. There exist solutions $v_{i}(\sigma, \tau, \zeta)$ of problems (21), (22) in the domain $\sigma^{4}+$ $\tau^{4} \leq \zeta$. These functions admit the asymptotic expansions (23) as $\zeta \rightarrow \infty$, and these expansions can be differentiated termwise with respect to all variables. 
Proof. First, we verify that the series (23) are asymptotic solutions of system (21), i.e., for large $\zeta$, the partial sums of these series satisfy system (21) approximately. Although this statement seems almost obvious, a rigorous proof of it is, unfortunately, very cumbersome. A natural way of proving this starts with writing $\mathbb{M}_{\varepsilon} \mathbb{A}_{m, \sigma, \tau, \zeta} \mathbb{A}_{N, x, y, z} U-f(x, y, z)$ in different variables. Since this value is small in some sense, from (24) it follows that $\mathbb{M}_{\varepsilon} \mathbb{A}_{N, x, y, z} \mathbb{A}_{m, \sigma, \tau, \zeta} \widetilde{V}-f(x, y, z)$ is also small. This implies that the series $\widetilde{v}_{i}(\sigma, \tau, \zeta)$ are asymptotic solutions of system (21).

We proceed to realizing this, unfortunately, very cumbersome and unpleasant plan. We have

$$
\mathbb{M}_{\varepsilon} \mathbb{A}_{N, x, y, z} U-f(x, y, z)=\mathbb{M}_{\varepsilon}\left(\sum_{k=0}^{N} \varepsilon^{k} u_{k}(x, y, z)\right)-f(x, y, z)=\varepsilon^{N+1} \frac{\partial^{2} u_{N}}{\partial z^{2}} .
$$

Let $\Gamma_{p}(\xi, \eta, z)$ and $\Gamma_{p q}(\xi, \eta, z)$ denote bounded functions that are smooth for $z>0$ and have asymptotic expansions in positive integral powers of $z^{\frac{1}{4}}$ as $z \rightarrow+0$. Thus,

$$
\mathbb{M}_{\varepsilon} \mathbb{A}_{N, x, y, z} U-f(x, y, z)=\varepsilon^{N+1} z^{\frac{-3 N-3}{2}} \Gamma_{00}(\xi, \eta, z)=\zeta^{\frac{-3 N-3}{2}} \Gamma_{00}(\xi, \eta, z) .
$$

For brevity, we denote by $\mathbb{B}_{m, N}$ the finite sums $\mathbb{A}_{m, \sigma, \tau, \zeta} \mathbb{A}_{N, x, y, z} U$ and $\mathbb{A}_{N, x, y, z} \mathbb{A}_{m, \sigma, \tau, \zeta} \tilde{V}$, which are equal by (24). Consequently,

$$
\begin{aligned}
\mathbb{M}_{\varepsilon} \mathbb{B}_{m, N}-f(x, y, z) & =\mathbb{M}_{\varepsilon} \mathbb{A}_{N, x, y, z} U-f(x, y, z)+\mathbb{M}_{\varepsilon}\left(\mathbb{B}_{m, N}-\mathbb{A}_{N, x, y, z} U\right) \\
& =\zeta^{\frac{-3 N-3}{2}} \Gamma_{00}(\xi, \eta, z)+\mathbb{M}_{\varepsilon}\left(\mathbb{B}_{m, N}-\mathbb{A}_{N, x, y, z} U\right) .
\end{aligned}
$$

Next, we transform the last term:

$$
\begin{aligned}
\mathbb{M}_{\varepsilon}\left(\mathbb{B}_{m, N}-\mathbb{A}_{N, x, y, z} U\right)=\mathbb{M}_{\varepsilon}\left(\sum_{k=0}^{N} \varepsilon^{k}\left[\sum_{j=6 m-6 k+1}^{\infty} z^{\frac{j}{4}} u_{k j}\left(\sigma \zeta^{-\frac{1}{4}}, \tau \zeta^{-\frac{1}{4}}\right)\right]\right) \\
=\sum_{k=0}^{N} \varepsilon^{k} \mathbb{M}_{\varepsilon}\left[z^{\frac{6 m-6 k+1}{4}} \sum_{j=0}^{\infty} z^{\frac{j}{4}} u_{k, 6 m-6 k+j+1}(\xi, \eta)\right] \\
=\sum_{k=0}^{N} \varepsilon^{k}\left[z^{\frac{6 m-6 k-1}{4}} \Gamma_{k, 1}(\xi, \eta, z)+\varepsilon z^{\frac{6 m-6 k+1}{4}-2} \Gamma_{k, 2}(\xi, \eta, z)\right] .
\end{aligned}
$$

Finally, we obtain

$$
\mathbb{M}_{\varepsilon} \mathbb{B}_{m, N}-f(x, y, z)=\zeta^{\frac{-3 N-3}{2}} \Gamma_{00}(\xi, \eta, z)+\sum_{k=0}^{N} \varepsilon^{m-\frac{1}{6}} \zeta^{\frac{3}{2}(m-k)-\frac{1}{4}} \Gamma_{k, 3}(\xi, \eta, z) .
$$

We take into account the fact that

$$
\mathbb{B}_{m, N}=\sum_{i=2}^{6 m} \varepsilon^{\frac{i}{6}} v_{i, N}(\sigma, t, \zeta)
$$

and represent the operator $\mathbb{M}_{\varepsilon}$ in the variables $\sigma, t$, and $\zeta$; then relation (26) shows that the functions $v_{i, N}(\sigma, t, \zeta)$ satisfy system (21) approximately. This means that the following relations are valid:

$$
\left\{\begin{array}{l}
\mathrm{M}_{0} v_{2, N}=Q_{0}(\sigma, \tau, \zeta)+O\left(\zeta^{-\frac{6 N+1}{4}}\right) \\
\mathrm{M}_{0} v_{3, N}=-\mathrm{M}_{1} v_{2, N}+Q_{1}(\sigma, \tau, \zeta)+O\left(\zeta^{-\frac{6 N+2}{4}}\right), \\
\cdots \\
\mathrm{M}_{0} v_{m, N}=-\sum_{j=1}^{m-2} \mathrm{M}_{j} v_{m-j, N}+Q_{m-2}(\sigma, \tau, \zeta)+O\left(\zeta^{-\frac{6 N+m-1}{4}}\right) .
\end{array}\right.
$$

Now, it remains to prove the existence of the above-mentioned functions $v_{i}(\sigma, \tau, \zeta)$. This follows from the next lemma. 
Lemma. Let $\Omega=\left\{(\sigma, \tau, \zeta): \sigma^{4}+\tau^{4} \leq \zeta\right\}, w_{i}(\sigma, \tau, \zeta) \in C^{\infty}(\Omega), w_{i}\left(\sigma, \eta, \sigma^{4}+\eta^{4}\right)=0$, and $w_{i}(\sigma, \tau, \zeta)=O\left(\zeta^{q-\frac{i}{6}}\right)$ as $\zeta \rightarrow \infty$. (Here, $q$ is a fixed number.) Suppose $\Phi(\sigma, \tau, \zeta) \in$ $C^{\infty}(\Omega)$, and let all derivatives of $\Phi(\sigma, \tau, \zeta)$ be functions of slow growth as $\zeta \rightarrow \infty$. Let the series $W^{*}=\sum_{i=0}^{\infty} w_{i}(\sigma, \tau, \zeta)$ be an asymptotic solution of the equation $\mathrm{M}_{0} W^{*}=$ $\Phi(\sigma, \tau, \zeta)$ in the domain $\Omega$ in the sense that for all positive integers $N$ we have

$$
\mathrm{M}_{0}\left(\sum_{i=0}^{N} w_{i}(\sigma, \tau, \zeta)\right)-\Phi(\sigma, \tau, \zeta)=O\left(\zeta^{q-\frac{N+4}{6}}\right)
$$

as $\zeta \rightarrow \infty$. Then the equation $\mathrm{M}_{0} W=\Phi(\sigma, \tau, \zeta)$ admits a solution $W(\sigma, \tau, \zeta) \in C^{\infty}(\Omega)$ for which $W^{*}$ is an asymptotic series as $\zeta \rightarrow \infty$.

Proof. Let $\Omega_{r}$ be the subset of $\Omega$ defined by the inequality $\zeta \leq r$, and let $W_{r}(\sigma, \tau, \zeta)$ be a solution of the equation $\mathrm{M}_{0} W_{r}=\Phi(\sigma, \tau, \zeta)$ that is zero on the boundary of this domain. By the assumptions of the lemma, there exist numbers $h>0$ and $K>0$ such that $|\Phi(\sigma, \tau, \zeta)|<K\left(1+\zeta^{h}\right)$.

First, we find a uniform estimate for the solutions $W_{r}(\sigma, \tau, \zeta)$ in $\Omega$. We consider the function

$$
H(\sigma, \tau, \zeta)=\left(2 \sqrt{\zeta+\alpha}-\sigma^{2}-\tau^{2}\right) \exp (\sqrt{\zeta+\alpha}), \quad \alpha>0
$$

positive in the domain $\Omega$. Since for a sufficiently large fixed $\alpha$ we have

$$
\begin{aligned}
\mathrm{M}_{0}(H) & <\left(\frac{1}{\zeta+\alpha}+\frac{1}{2 \sqrt{\zeta+\alpha}}-2 a_{11}(0,0,0)-2 a_{22}(0,0,0)\right) \exp (\sqrt{\zeta+\alpha}) \\
& <-\gamma \exp (\sqrt{\zeta+\alpha}), \quad \gamma>0,
\end{aligned}
$$

we see that

$$
\mathrm{M}_{0}\left(C H(\sigma, \tau, \zeta) \pm W_{r}(\sigma, \tau, \zeta)\right)<0
$$

for some positive $C$.

The maximum principle shows that $\left|W_{r}(\sigma, \tau, \zeta)\right|<C H(\sigma, \tau, \zeta)$. Consequently, the functions $W_{r}(\sigma, \tau, \zeta)$ are uniformly bounded on each compact subset of $\Omega$. From the wellknown estimates [14, 15] for the derivatives of solutions of elliptic equations, it follows that the derivatives of these solutions are uniformly bounded on any fixed compact set. Choosing a subsequence of solutions $W_{r}(\sigma, \tau, \zeta)$ that converges on this compact set, we obtain a solution of the equation $\mathrm{M}_{0} W_{r}=\Phi(\sigma, \tau, \zeta)$ on the same compact set. Next, extending the compact set and choosing a diagonal sequence in a standard way, we obtain a solution $W(\sigma, \tau, \zeta)$ of the equation in question in the entire domain $\Omega$. For this solution, we have the estimate $|W(\sigma, \tau, \zeta)|<C H(\sigma, \tau, \zeta)$.

Now, we must verify that the solution constructed above has the asymptotic expansion $\sum_{i=0}^{\infty} w_{i}(\sigma, \tau, \zeta)$ as $\zeta \rightarrow \infty$.

For this, we consider the difference $g_{N}=\sum_{i=0}^{N} w_{i}(\sigma, \tau, \zeta)-W(\sigma, \tau, \zeta)$. By the assumptions of the lemma, we have $\left|\mathrm{M}_{0} g_{N}\right|<\gamma(1+\zeta)^{-\frac{N+4}{6}}$ in $\Omega$. As above, we can use the barrier function $\left(2 \sqrt{\zeta+\alpha}-\sigma^{2}-\tau^{2}\right)(1+\zeta)^{-\frac{N+4}{6}}$ to show that $g_{N}=O(1+\zeta)^{-\frac{N+4}{6}}$. (We note that the maximum principle is valid in the unbounded domain $\Omega$. This can be proved with the help of the same function $H(\sigma, \tau, \zeta)$, because we have an estimate for the function $W(\sigma, \tau, \zeta)$ and the $w_{i}(\sigma, \tau, \zeta)$ are functions of slow growth.) The lemma is proved.

Now, we can finish the proof of Theorem 2 by applying the above lemma to systems (21) and (27). We start with the first equations of the systems. The lemma cannot be applied to the function $v_{2, N}$ directly, because, in general, the terms of its partial sum (23) have singularities as $\zeta \rightarrow 0$. However, this does not play any essential role because the functions $u_{k, i-6 k}\left(\sigma \zeta^{-\frac{1}{4}}, \tau \zeta^{-\frac{1}{4}}\right)$ can be replaced by the same functions multiplied by a fixed 
smooth cutoff function equal to zero in a neighborhood of zero and equal to 1 for $\zeta>1$. This change does not affect the asymptotic properties as $\zeta \rightarrow \infty$. Therefore, the lemma implies the existence of a solution $v_{2}(\sigma, \tau, \zeta)$ with an asymptotic expansion $\widetilde{v}_{2}(\sigma, \tau, \zeta)$ of the form (23). Next, we must pass to the functions $v_{3}(\sigma, \tau, \zeta)$ and $\widetilde{v}_{3}(\sigma, \tau, \zeta)$. Applying the lemma to the equations for these functions in systems (21) and (27), we arrive at the required solution $v_{3}(\sigma, \tau, \zeta)$. Proceeding by induction, we obtain the conclusion of Theorem 2.

Thus, we have constructed an external expansion (3) in the domain $D$ and an internal expansion (20) in a neighborhood of the point $\mathbb{P}_{0}=(0,0,0)$. We assume that in a neighborhood of the other singular point $\mathbb{P}_{1}=\left(x_{1}, y_{1}, z_{1}\right), z_{1}>0$, the boundary has an equation of a similar form $z_{1}-z=\left(x-x_{1}\right)^{4}+\left(y-y_{1}\right)^{4}$ and construct a similar internal expansion, which we denote by $V^{*}$, in a neighborhood of this point.

Theorem 3. As $\varepsilon \rightarrow 0$ and for $\varepsilon^{\frac{1}{3}} \leq z \leq z_{1}-\varepsilon^{\frac{1}{3}}$, the solution of problem (11), (2) admits the asymptotic expansion (3). This solution can be expanded into the uniform asymptotic series (20) for $0 \leq z \leq \varepsilon^{\frac{1}{3}}$ and into the uniform asymptotic series $V^{*}$ for $z_{1}-\varepsilon^{\frac{1}{3}} \leq z \leq z_{1}$.

Proof. By the construction of the series (3) and formula (25), the function $\mathbb{A}_{N, x, y, z} U$ satisfies the boundary condition (2) and equation (11) up to $\varepsilon^{\frac{N}{2}}$ for $z \geq \varepsilon^{\frac{1}{3}}$. On the other hand, by the construction of the series (20), the function $\mathbb{A}_{m, \sigma, \tau, \zeta} V$ satisfies the boundary condition (2) and equation (1) up to $\varepsilon^{\frac{m}{2}}$ for $z \leq \varepsilon^{\frac{1}{3}}$. Moreover, the form of the asymptotic series (12) shows that

$$
\left|\mathbb{A}_{N, x, y, z} U-\mathbb{B}_{m, N}\right|<K \varepsilon^{\frac{N}{2}}
$$

whenever $z \leq \varepsilon^{\frac{1}{3}}$, and the form of the asymptotic series (23) shows that

$$
\left|\mathbb{A}_{m, \sigma, \tau, \zeta} V-\mathbb{B}_{m, N}\right|<K \varepsilon^{\frac{N}{2}}
$$

whenever $z \geq \varepsilon^{\frac{1}{3}}$.

Thus, the function

$$
\mathbb{E}_{m n}=\mathbb{A}_{N, x, y, z} U+\mathbb{A}_{m, \sigma, \tau, \zeta} V-\mathbb{B}_{m, N}
$$

satisfies the equation approximately for $0<z<\delta$, where $\delta$ is a fixed small positive number. Indeed, for $\varepsilon^{\frac{1}{3}} \leq z<\delta$, we have

$$
\begin{aligned}
\left|\mathbb{M}_{\varepsilon} \mathbb{E}_{m n}-f(x, y, z)\right| & =\left|\mathbb{M}_{\varepsilon} \mathbb{A}_{N, x, y, z} U-f(x, y, z)+\mathbb{M}_{\varepsilon}\left[\mathbb{A}_{m, \sigma, \tau, \zeta} V-\mathbb{B}_{m, N}\right]\right| \\
& <K_{1}\left(\varepsilon^{\frac{N}{2}}+\varepsilon^{\frac{m}{2}}\right) .
\end{aligned}
$$

On the other hand, if $z \leq \varepsilon^{\frac{1}{3}}$, then

$$
\begin{aligned}
\left|\mathbb{M}_{\varepsilon} \mathbb{E}_{m n}-f(x, y, z)\right| & =\left|\mathbb{M}_{\varepsilon} \mathbb{A}_{m, \sigma, \tau, \zeta} V-f(x, y, z)+\mathbb{M}_{\varepsilon}\left[\mathbb{A}_{N, x, y, z} U-\mathbb{B}_{m, N}\right]\right| \\
& <K_{2}\left(\varepsilon^{\frac{m}{2}}+\varepsilon^{\frac{N}{2}}\right) .
\end{aligned}
$$

As was noted above, in a neighborhood of the point $\mathbb{P}_{1}=\left(x_{1}, y_{1}, z_{1}\right)$ we construct an internal expansion $V^{*}$. As in the case of the point $\mathbb{P}_{0}=(0,0,0)$, we define $\mathbb{B}_{m, N}^{*}$ as the common part of the asymptotic expansions of the series (3) and $V^{*}$. For these series we have the same estimates as for the series in a neighborhood of the point $\mathbb{P}_{0}$.

The terms of the series $V$ and $V^{*}$ are defined only in neighborhoods of the points $\mathbb{P}_{0}$ and $\mathbb{P}_{1}$. Therefore, to finish the proof rigorously, we must multiply the terms of these series by smooth cutoff functions. One of the functions is identically 1 for $z \leq \delta$ and zero for $z \geq 2 \delta$. The other function is constructed similarly for $z \leq z_{1}$. To avoid superfluous 
notation, we preserve the old notation for the new functions. It is obvious that the asymptotic properties of the functions $v_{k}(\sigma, \tau, \zeta)$ and $\widetilde{v}_{k}(\sigma, \tau, \zeta)$ will not change.

Thus, the function

$$
\mathbb{E}_{m n}^{*}=\mathbb{A}_{N, x, y, z} U+\mathbb{A}_{m, \sigma, \tau, \zeta} V+\mathbb{A}_{m, \sigma, \tau, \zeta} V^{*}-\mathbb{B}_{m, N}-\mathbb{B}_{m, N}^{*}
$$

is defined everywhere in the domain $D$ and satisfies the boundary condition (2) and the estimate

$$
\left|\mathbb{M}_{\varepsilon}\left(\mathbb{E}_{m N}^{*}-u_{\varepsilon}(x, y, z)\right)\right|<K_{3}\left(\varepsilon^{\frac{m}{2}}+\varepsilon^{\frac{N}{2}}\right)
$$

Consequently,

$$
\left|\left(\mathbb{E}_{m N}^{*}-u_{\varepsilon}(x, y, z)\right)\right|<K_{4}\left(\varepsilon^{\frac{m}{2}}+\varepsilon^{\frac{N}{2}}\right) .
$$

Now, the claim of Theorem 3 follows from the above estimate and inequalities (28) and (29).

Remark 1. For simplicity, the boundaries of the neighborhoods under consideration were chosen to be $z=\varepsilon^{\frac{1}{3}}$ and $z=z_{1}-\varepsilon^{\frac{1}{3}}$. Without any serious changes in the above proof, we can replace them by $z=\varepsilon^{\alpha}$ and $z=z_{1}-\varepsilon^{\alpha}$ provided that $0<\alpha<\frac{2}{3}$.

Remark 2. Here we point out an interesting specific feature of the problem in question. We assumed above that, in a neighborhood of the origin, the boundary has the equation $z=x^{4}+y^{4}$, and, in this case, the asymptotic expansion is invalid in a small neighborhood of the origin. The coefficients of it have growing singularities as $z \rightarrow+0$. It is easy to verify that the same is true if the curvature of the boundary is zero at the points where the boundary touches the plane $z=$ const. At the same time, if the curvature is nonzero, then the coefficients of the external asymptotic expansion have no singularities. Thus, the problem remains singular but not bisingular.

As an example, we consider the specific case of problem (1), (2) in which, for $z<\delta$, the domain $D$ coincides with the interior of the paraboloid of revolution $z=x^{2}+y^{2}$ and, in a neighborhood of the origin, the principal part of the operator $\mathrm{L}$ coincides with the Laplace operator, i.e., $\mathrm{L}_{0}=\frac{\partial^{2}}{\partial \xi^{2}}+\frac{\partial^{2}}{\partial \eta^{2}}$ in formula (10).

Now, we state some auxiliary results. We denote by $U_{k}^{(j)}(x, y), j=1,2$, the harmonic polynomials (in the variables $x$ and $y$ )

$$
U_{k}^{(1)}(x, y)=r^{k} \sin k \phi, \quad U_{k}^{(2)}(x, y)=r^{k} \cos k \phi,
$$

where $r$ and $\phi$ are polar coordinates in the plane $(x, y)$. It is easy to verify the relations

$$
\begin{aligned}
& x U_{k}^{(1)}=\frac{1}{2} U_{k+1}^{(1)}+\frac{1}{2} r^{2} U_{k-1}^{(1)}, \quad k \geq 2, \quad x U_{k}^{(2)}=\frac{1}{2} U_{k+1}^{(2)}+\frac{1}{2} r^{2} U_{k-1}^{(2)}, \quad k \geq 1, \\
& y U_{k}^{(1)}=\frac{1}{2} r^{2} U_{k-1}^{(2)}-\frac{1}{2} U_{k+1}^{(2)}, \quad k \geq 1, \quad y U_{k}^{(2)}=\frac{1}{2} U_{k+1}^{(1)}+\frac{1}{2} r^{2} U_{k-1}^{(1)}, \quad k \geq 2,
\end{aligned}
$$

whence it follows that the function $x^{m} y^{p} U_{k}^{(j)}$ is a linear combination of the functions $U_{k+m+p-2 s}^{(j)} r^{2 s}, 0 \leq s \leq \frac{k+m+p}{2}$, for all $m, p \geq 0$.

Consequently ( since $U_{0}^{(2)}=1, U_{1}^{(2)}=x$, and $U_{1}^{(1)}=y$ ), every polynomial $Q(x, y)$ can be represented as a linear combination of functions of the form $r^{2 q} U_{k}^{(j)}$.

It is also easily seen that

$$
\Delta\left(r^{\alpha} U_{k}^{(j)}\right)=[\alpha(\alpha-1)+2 \alpha k+\alpha] r^{\alpha-2} U_{k}^{(j)}=\alpha(\alpha+2 k) r^{\alpha-2} U_{k}^{(j)}
$$

for all $\alpha$. Therefore, the function

$$
w(x, y)=\frac{1}{(2 n+2)(2 n+2+2 k)} r^{2 n+2} U_{k}^{(j)},
$$


which is a homogeneous polynomial of degree $2 n+k+2$, is a solution of the nonhomogeneous equation

$$
\Delta_{x y} w=r^{2 n} U_{k}^{(j)} .
$$

On the boundary of the disk $\Omega_{z}$ (for $r=\sqrt{z}$ ), the function constructed above coincides with the harmonic polynomial $U_{k}^{(j)}(x, y)$ up to the factor $z^{n+1}[(2 n+2)(2 n+2+2 k)]^{-1}$. Therefore, the function

$$
\widetilde{w}(x, y, z)=\frac{1}{(2 n+2)(2 n+2+2 k)}\left[r^{2 n+2}-z^{n+1}\right] U_{k}^{(j)}
$$

is a unique bounded solution of the problem

$$
\Delta_{x y} w=r^{2 n} U_{k}^{(j)}, \quad r<\sqrt{z} ; \quad w(\sqrt{z}, \phi)=0 .
$$

By construction, this solution is a polynomial in all three variables $x, y$, and $z$.

Using the above constructions, the structure of the operator $\mathrm{L}$ in a neighborhood of the origin, and also the form of the right-hand sides of relations (4), we can easily show that, for $z \leq x^{2}+y^{2}$, the asymptotic expansions of the solutions $u_{k}(x, y, z)$ as $z \rightarrow 0$ are series, the terms of which are polynomials of an appropriate structure in all three variables $x, y, z$.

Consequently, in this particular case, the coefficients of the external expansion (3) have no singularities, there is no need to introduce the external extension, and the series (3) provides the uniform asymptotic approximation of the solution $u_{\varepsilon}(x, y, z)$ as $\varepsilon \rightarrow 0$.

\section{REFERENCES}

[1] N. Levinson, The first boundary value problem for $\varepsilon \Delta u+A(x, y) u_{x}+B(x, y) u_{y}+C(x, y) u=f(x, y)$ for small $\varepsilon$, Ann. of Math. (2) $\mathbf{5 1}$ (1950), 428-445. MR0033433(11:439k)

[2] O. A. Oleǐnik, On equations of elliptic type with a small parameter in the highest derivatives, Mat. Sb. (N. S.) 31 (1952), no. 1, 104-117. (Russian) MR0052012 (14:560b)

[3] S. L. Kamenomostskaya, The first boundary problem for equations of elliptic type with a small parameter with the highest derivatives, Izv. Akad. Nauk SSSR Ser. Mat. 19 (1955), no. 5, 345-360. (Russian) MR0074667 (17:627a)

[4] M. I. Vishik and L. A. Lyusternik, Regular degeneration and boundary layer for linear differential equations with small parameter, Uspekhi Mat. Nauk 12 (1957), no. 5, 3-122. (Russian) MR0096041 (20:2539)

[5] V. A. Trenogin, The development and applications of the Lyusternik-Vishik asymptotic method, Uspekhi Mat. Nauk 25 (1970), no. 4, 123-156; English transl. in Russian Math. Surveys 25 (1970), no. 4. MR0274921 (43:679)

[6] S. Kaplun and P. A. Lagerstrom, Asymptotic expansions of Navier-Stokes solutions for small Reynolds numbers, J. Math. Mech. 6 (1957), 585-593. MR0091693 (19:1004f)

[7] M. Van Dyke, Perturbation methods in fluid mechanics, Appl. Math. Mech., vol. 8, Acad. Press, New York-London, 1964. MR0176702 (31:974)

[8] V. M. Babich and N. Ya. Kirpichnikova, The boundary layer method in diffraction problems, Leningrad. Univ., Leningrad, 1974; English transl., Springer Ser. in Electrophys., vol. 3, SpringerVerlag, Berlin-New York, 1979. MR0481585 (58:1697); MR0555574 (82b:35013)

[9] A. M. Il'in and E. F. Lelikova, The method of matching asymptotic expansions for the equation $\varepsilon \Delta u-a(x, y) u_{y}=f(x, y)$ in a rectangle, Mat. Sb. (N. S.) 96 (1975), no. 4, 568-583; English transl. in Math. USSR-Sb. 25 (1975), no. 4. MR0382824(52:3706)

[10] A. M. Il'in, Matching of asymptotic expansions of solutions of boundary value problems, Nauka, Moscow, 1989; English transl., Transl. Math. Monogr., vol. 102, Amer. Math. Soc., Providence, RI, 1992. MR 1007834 (90i:35062), MR1182791 (93g:35016)

[11] E. F. Lelikova, On the asymptotic behavior of a solution of a second-order elliptic equation with small parameter at a highest derivative, Trudy Inst. Mat. Mekh. Ural. Otdel. Ross. Akad. Nauk 9 (2003), no. 1, 107-119. (Russian)

[12] _ On the asymptotic behavior of the solution of an equation with a small parameter, Dokl. Akad. Nauk 429 (2009), no. 4, 447-450. (Russian) MR2649174 
[13] _ On the asymptotic behavior of the solution of a second-order elliptic equation with small parameter at a highest derivative, Trudy Moskov. Mat. Obshch. 71 (2010), 162-199; English transl., Trans. Mosc. Math. Soc. 71 (2010), 141-174.

[14] O. A. Ladyzhenskaya and N. N. Ural'tseva, Linear and quasilinear equations of elliptic type, Nauka, Moscow, 1964; English transl., Acad. Press, New York-London, 1968. MR0211073 (35:1955) MR0244627 (39:5941)

[15] L. Bers, F. John, and M. Schechter, Partial differential equations, Lectures in Appl. Math., vol. 3A, Amer. Math. Soc., Providence, RI, 1979. MR0598466 (82c:35001)

Chelyabinsk State University, Ul. Brat'yev Kashirinyh 129, Chelyabinsk 454001, Russia

E-mail address: iam@csu.ru

Institute of Mathematics and Mechanics, of Ural Branch of Russian Academy of Science, Ul. Sof'i Kovalevskoi 16, Ekaterinburg 620990, Russia

E-mail address: lef@imm.uran.ru

Received 11/JUN/2010

Translated by B. M. BEKKER 